\title{
Nonstrategic factors underlie improvements in free recall during middle childhood
}

\author{
MARGARET B. TINZMANN and JAMES W. HALL \\ Northwestern University, Evanston, Illinois
}

\begin{abstract}
Free recall of Grade 4 and Grade 8 children was examined under five presentation conditions that varied in their amenability to strategy use. No interaction suggesting an important role for changes in strategic (deliberate) rehearsal was found, suggesting that increases in free recall during middle childhood chiefly derive from nonstrategic processing during study.
\end{abstract}

In previous experiments (Hall \& Tinzmann, 1985, 1989), we found that increases in free recall from about age 7 to age 12 were as great when presentation rates were fast, presumably restricting the use of strategic rehearsal, as when rates were sufficiently slow for such rehearsal to occur. These results suggest that changes in rehearsal strategies do not underlie differences in the free recall of unrelated words over that age range.

In the present experiment, we have delved further into the question of study-strategy differences between children in Grades 4 and 8, by comparing the effects of five presentation conditions that differed in their amenability to strategy use. Three of the conditions varied in the rate and frequency with which study lists were presented; the lists were presented four times at a 1.2-sec rate, twice at a 2.4-sec rate, or once at a 4.8-sec rate. In the fourth (the self-paced) condition, the children were given control over presentation durations. In the complete condition, the entire list was shown on the screen for the total period of study.

The relative importance of changes in rehearsal strategies may be estimated from the relative effectiveness of the above conditions for recall at the two grade levels. The 1.2-sec condition involved the imposition of a study procedure (spaced repetitions) well known to be effective for recall, while at the same time it restricted the subjects' opportunities for strategic (self-initiated) study activities. Thus, not only would the $1.2-\mathrm{sec}$ condition be expected to yield relatively good recall, but to the extent that age differences in recall are a result of differences in spontaneous strategy implementation, age differences in recall should be quite small in the 1.2-sec condition. The absolute recall levels in the 4.8-sec condition should be lower, and, from the strategy-development perspec-

This experiment was a portion of the doctoral research of the first author who now is at the North Central Regional Educational Laboratory, Elmhurst, IL 60126. We thank Karen Fuson, Dan Hall, and the cooperating school officials and students in the Mt. Prospect elementary schools. Reprint requests should be addressed to James W. Hall, Department of Psychology, Northwestern University, Evanston, IL 60208. tive, especially so for the Grade 4 children; and the more pronounced the interaction, the greater the importance of increased strategy use. The failure to observe the interaction at all would imply that increased strategy use is not an important factor in the recall increases found across this age range.

This kind of analysis may be extended to the complete and self-paced conditions, so that the developmental importance of strategies permitted by these conditions may be estimated from the relative effectiveness of the conditions at the two grade levels. To illustrate: in several experiments with adults, complete list presentation has proven superior to discrete presentation (e.g., Hall, Cox, \& Tinzmann, 1984; Hall, Smith, Wegener, \& Underwood, 1981), presumably because of the greater degree to which complete presentation facilitates strategic study activities, including the review of earlier items, as the subject proceeds through the list. If older children are more strategic than younger ones in this respect, one would expect the sort of interaction between grade-level and presentation method (discrete vs. complete presentation) that was described above.

An important difference between this experiment and earlier ones was that an attempt was made here to equate the difficulty level of the recall task for the two age levels. This was done because a true interaction could possibly be missed, or a spurious one observed, because of a scale (difficulty level) difference at the two age levels. If recall levels for the two age groups were substantially different, as might be expected if identical lists and procedures were to be used, the interpretation of an interaction involving age would be less clear than if difficulty levels were similar (see, for example, the discussion of this issue by Baron \& Trieman, 1980). To achieve the desired similarity of difficulty levels, we varied the lists' lengths: the Grade 8 children received 14-word lists and the Grade 4 children were given 9 -word lists. This, of course, eliminated the possibility of evaluating a main effect of age. However, we had no interest in demonstrating once again that older children recall more words than do younger ones. Our interests were entirely in the effects of presentation condition and especially in any possible interactions between age and presentation condition. 


\section{METHOD}

\section{Subjects and Design}

The subjects were 80 children from Grade 4 and 80 from Grade 8, in a middle-class Chicago suburb. They ranged in years from 8.6 to 10.0 (mean $=9.4$ ) at Grade 4 , and from 12.4 to 14.1 at Grade 8 (mean $=13.3$ ). Children identified as learning disabled or poor in reading were excluded. At each grade level, 16 children were assigned randomly to each of the five experimental conditions. Each subject studied, and was tested for free recall on, three word lists in one of five conditions that differed in the details of list presentation but were identical in total study time per item. The first list functioned to familiarize the subjects with the materials and procedures. The lists were the same for each presentation condition, and they were presented in the same order across conditions.

In three of the conditions, presentation was discrete (the usual serial presentation), and the presentation rate was controlled by means of an Apple II microcomputer. In the discrete slow condition, each list was presented once at the rate of $4.8 \mathrm{sec}$ per item. In the discrete medium condition, each list was presented two times at a $2.4-\mathrm{sec}$ rate. In the discrete fast condition, each list was presented four times at a 1.2-sec rate. In the self-paced condition, the items were also displayed serially, but the subjects controlled the presentation durations by pressing the space bar on the computer keyboard. In the complete condition, the entire list was displayed on the computer screen. In the conditions in which the lists were presented more than once, the order of presentation was the same each time.

Two children in Grade 4 and four in Grade 8 in the self-paced condition were replaced, because they selected presentation rates that were so slow that they did not view each entire list.

\section{Materials and Procedure}

The lists were made up from a pool of $\mathbf{4 2}$ highly familiar one-syllable nouns (e.g., BIRD, COW, FRUIT, MOON). These were assigned to the three lists randomly, except that words that appeared to be strongly associated with each other were not placed in the same list. The resulting 14-word lists were used with the Grade 8 subjects. The 9-word lists used with the Grade 4 subjects were formed by means of randomly removing 5 of the words from each of the 14-word lists. Preliminary work had indicated that these differences in list length would result in roughly similar percentages of recall at the two grade levels.

The subjects were seen individually in one 10 - to 15 -min session in a separate room within their school. The session began with an explanation of the task, followed by the first (familiarization) list, in accordance with the subject's presentation condition. The two critical experimental lists were then presented in the same way. Each list presentation was preceded by the brief display of an asterisk at the center of the screen. In all but the complete condition, each word appeared at the center of the screen; in the complete condition, the words appeared in a single column on the screen. Immediately after study, a fourdigit number appeared for approximately $3 \mathrm{sec}$, and the subjects were instructed to remember that number and say it at the beginning of the recall period. Thus, the number served both to signal the end of the study phase and to eliminate strong recency effects due to words' remaining in working memory at the beginning of the recall period. Recall was oral, with no time limit.

\section{RESULTS AND DISCUSSION}

Our manipulation of list length resulted in similar task difficulty for the two age levels. Averaged over presentation conditions and lists, the mean proportions correct were .53 for Grade 4 and .52 for Grade 8 . This set the stage for a straightforward interpretation of any observed interactions or of the absence of such interactions. The various analyses were applied to recall scores summed across the two experimental lists within each condition and expressed as proportions.
For both grades, recall was markedly lower with a single 4.8-sec presentation than in the other conditions (see Table 1). There was little difference among the remaining conditions and no indication of an interaction between age and presentation condition. These observations were confirmed by statistical analyses. A 5 (presentation conditions) $\times 2$ (age) analysis of variance yielded a significant main effect of presentation conditions $[F(4,150)=$ $523, M S_{\mathrm{e}}=1.88$ ]; the interaction did not approach significance $(F<1)$. Individual comparisons, using the Newman-Keuls procedure, confirmed that the $4.8-\mathrm{sec}$ condition differed reliably from each of the other conditions, and that no other differences were significant. In short, as in our earlier experiments, there was no evidence here for changes in rehearsal strategies as an important source of increases in free recall across middle childhood.

We checked the possibility that for the self-paced condition there would be an age difference in pacing that might indicate a strategic difference. The older children did select somewhat briefer presentation times (the mean exposure time per item was $1.87 \mathrm{sec}$ at Grade 8 and $2.63 \mathrm{sec}$ at Grade 4); but that difference was not significant, no doubt partly attributable to differences in reading speed, even though poor readers within each grade level were excluded from the sample.

Our findings and conclusions are consistent with other proposals to the effect that nonstrategic factors involving semantic memory (knowledge) changes have been underestimated in strategy-oriented explanations of memory development (e.g., Bjorklund, 1985; Wilkinson, 1981). The ways in which both strategic and nonstrategic changes work to affect memory development are not fully understood, but for free recall it is plausible (and has been suggested by Ackerman, 1986) that developmental changes in recall involve increases in the amount of relational information that is encoded, where relational information consists of associations among list items, as well as associations between such items and elements of the experimental context (see Hunt \& Einstein, 1981). Such associations allow list words that are retrieved during testing, and contextual elements present at both study and testing, to serve as effective retrieval cues. One would expect increasing generation of relational information during study as a consequence of age-related increases in the richness of semantic memory (i.e., verbal knowledge). Furthermore, this generation may be expected to occur independently of whether the associative networks in-

Table 1

Means and Standard Deviations of Recall Proportions

\begin{tabular}{|c|c|c|c|c|c|c|c|c|c|c|}
\hline \multirow[b]{2}{*}{ Grade Level } & \multicolumn{2}{|c|}{$\begin{array}{l}\text { Discrete } \\
\text { Slow }\end{array}$} & \multicolumn{2}{|c|}{$\begin{array}{l}\text { Discrete } \\
\text { Medium }\end{array}$} & \multicolumn{2}{|c|}{$\begin{array}{l}\text { Discrete } \\
\text { Fast }\end{array}$} & \multicolumn{2}{|c|}{ Self-Paced } & \multicolumn{2}{|c|}{ Complete } \\
\hline & $M$ & $S D$ & $M$ & $S D$ & $M$ & $S D$ & $M$ & $S D$ & $M$ & $S D$ \\
\hline 4 & .47 & .15 & .56 & .10 & .54 & 1 & .52 & .1 & .58 & .19 \\
\hline 8 & .40 & .12 & .50 & .12 & .58 & .13 & .52 & .14 & .58 & .13 \\
\hline
\end{tabular}

Note-Discrete slow presentation $=$ one list presentation at a 4.8 -sec rate; discrete medium $=$ two presentations at a $2.4-\mathrm{sec}$ rate, and discrete fast $=$ four presentations at a $1.2-\mathrm{sec}$ rate. 
volved are activated by subject-controlled rehearsal or by the perceptual operations that occur before such rehearsal is initiated.

\section{REFERENCES}

Ackerman, B. P. (1986). The use of item-specific and relational episodic information in the recall of children and adults. Journal of Experimental Child Psychology, 42, 115-143.

Baron, J., \& Treiman, R. (1980). Some problems in the study of differences in cognitive processes. Memory \& Cognition, 8, 313-321.

BjorKLUND, D. F. (1985). The role of conceptual knowledge in the development of organization in children's memory. In C. J. Brainerd \& M. Pressley (Eds.), Basic processes in memory development: Progress in cognitive development research (pp. 103-143). New York: Springer-Verlag.

Hall, J. W., Cox, B. E., \& Tinzmann, M. B. (1984). Recall of categorized and unrelated lists with complete versus discrete presentation and fast versus moderate presentation rates. Bulletin of the Psychonomic Society, 22, 398-400.

Hall, J. W., Smith, T. A., Wegener, S. L., \& Underwood, B. J. (1981). Rate and frequency as determinants of learning with complete and discrete list presentation. Memory \& Cognition, 9, 360-367.

Hall, J. W., \& TinzmanN, M. B. (1985). Presentation-rate effects and age differences in children's free recall. Bulletin of the Psychonomic Society, 23, 227-229.

Hall, J. W., \& TinzmanN, M. B. (1989). Sources of improved recall during the school years. Bulletin of the Psychonomic Society, 27, 315-316.

Hunt, R. R., \& EINSTEIN, G. O. (1981). Relational and item-specific information in memory. Journal of Verbal Learning \& Verbal Behavior, 20, 497-514.

WILKINSON, A. C. (1981). Growth functions for rapid remembering Journal of Experimental Child Psychology, 32, 354-371.

(Manuscript received November 16, 1988.) 